\title{
The McShane and the weak McShane integrals of Banach space-valued functions defined on $R^{m}$
}

Guoju Ye and Stefan Schwabik 


\title{
THE McSHANE AND THE WEAK McSHANE INTEGRALS OF BANACH SPACE-VALUED FUNCTIONS DEFINED ON $R^{m}$
}

\author{
Guoju Ye \\ Northwest Normal University \\ Lanzhou, People's Republic of China \\ yeguoju@math.cas.cz \\ STEFAN SCHWABIK \\ Matematický ústav AV ČR \\ Žitná 25, 11567 Praha 1, Czech Republic \\ schwabik@math.cas.cz
}

[Received October 13, 2001]

\begin{abstract}
In this paper, we define a concept of the weak McShane integral for functions mapping a compact interval $I_{0}$ in $R^{m}$ into a Banach space $X$ and discuss the relation between the weak McShane integral and the Pettis integral. We show that the weak McShane integral and the Pettis integral are equivalent if and only if the Banach space $X$ contains no copy of $c_{0}$. Further, combining the properties of the McShane integral and Pettis integral, we get some equivalent statements concerning the McShane integral and the Pettis integral.
\end{abstract}

Mathematical Subject Classification: 28B05, 46G10, 26A39

Keywords: Pettis integral, weak McShane integral, McShane integral

\section{Introduction}

It is known to all that the McShane integral of real-valued functions is a Riemann-type integral, which is equivalent to the Lebesgue integral. R. A. Gordon [1] generalized the definition of the McShane integral for real-valued functions to abstract functions from intervals in $R$ to Banach spaces and discussed some of its properties. In [2] it was proved that the McShane integral and Pettis integral are equivalent e.g. when the Banach space $X$ is reflexive with the property $(\mathrm{P})$.

In this paper, at first we get some equivalent statements about the Pettis and the McShane integrability of functions mapping an $m$-dimensional compact interval $I_{0}$ into a Banach space using the properties of the Pettis integral and McShane integral appearing in [3], [4], [5], [6], [2] and [7]. Then, we define a concept of the weak McShane integral and show that the weak McShane integral and the Pettis integral are equivalent if and only if the Banach space $X$ contains no copy of $c_{0}$. 


\section{The McShane integral}

We begin with some terminology and notations. We denote by $X$ a real Banach space with the norm $\|\cdot\|$ and $X^{*}$ its dual.

$I_{0}$ is a compact interval in $R^{m}, \Sigma$ is the set of all $\mu$-measurable subsets of $I_{0}, \mu$ stands for the Lebesgue measure.

$B\left(X^{*}\right)=\left\{x^{*} \in X^{*} ;\left\|x^{*}\right\| \leq 1\right\}$ is the closed unit ball in $X^{*}$.

We first extend the notion of partition of an interval. A partial $M$-partition $D$ in $I_{0}$ is a finite collection of interval-point pairs $(I, \xi)$ with non-overlapping intervals $I \subset I_{0}$, $\xi \in I_{0}$ being the associated point of $I$. Requiring $\xi \in I$ for the associated point of $I$, we get the concept of a partial $K$-partition $D$ in $I_{0}$. We write $D=\{(I, \xi)\}$.

A partial $M$-partition $D=\{(I, \xi)\}$ in $I_{0}$ is a $M$-partition of $I_{0}$ if the union of all the intervals $I$ equals $I_{0}$ and similarly for a $K$-partition.

Let $\delta$ be a positive function defined on the interval $I_{0}$. A partial $M$-partition (K-partition) $D=\{(I, \xi)\}$ is said to be $\delta$-fine if for each interval-point pair $(I, \xi) \in D$ we have $I \subset B(\xi, \delta(\xi))$ where $B(\xi, \delta(\xi))=\left\{t \in R^{m}\right.$; $\left.\operatorname{dist}(\xi, t)<\delta(\xi)\right\}$ and dist is the metric in $R^{m}$.

The $m$-dimensional volume of a given interval $I \subset I_{0}$ is denoted by $\mu(I)$.

Given a $M$-partition $D=\{(I, \xi)\}$ we write

$$
f(D)=(D) \sum f(\xi) \mu(I)
$$

for integral sums over $D$, whenever $f: I_{0} \mapsto X$.

DEFINITION 1. An $X$-valued function $f$ is said to be McShane integrable on $I_{0}$ if there exists an $S_{f} \in X$ such that for every $\varepsilon>0$, there exists $\delta(t)>0, t \in I_{0}$ such that for every $\delta$-fine $M$-partition $D=\{(I, \xi)\}$ of $I_{0}$, we have

$$
\left\|(D) \sum f(\xi) \mu(I)-S_{f}\right\|<\varepsilon \text {. }
$$

We write $(M) \int_{I_{0}} f=S_{f}$ and $S_{f}$ is the McShane integral of $f$ over $I_{0}$.

$f$ is McShane integrable on a set $E \subset I_{0}$ if the function $f \cdot \chi_{E}$ is McShane integrable on $I_{0}$, where $\chi_{E}$ denotes the characteristic function of $E$.

We write $(M) \int_{E} f=(M) \int_{I_{0}} f \chi_{E}=F(E)$ for the McShane integral of $f$ on $E$.

Denote the set of all McShane integrable functions $f: I_{0} \mapsto X$ by $\mathcal{M}$.

Replacing the term " $M$-partition" by " $K$-partition" in the definition above, we obtain Kurzweil-Henstock integrability and the definition of the Kurzweil-Henstock integral $(K) \int_{I_{0}} f$.

It is clear that if $f: I_{0} \mapsto X$ is McShane integrable, then it is also KurzweilHenstock integrable. 
The basic properties of the McShane integral, for example, linearity and additivity with respect to intervals can be found in [1-2], [5-6], [8], [9]. We do not present them here. The reader is referred to the above mentioned references for the details.

DEFINITION 2. A set $K \subset \mathcal{M}$ is called $M$-equiintegrable (McShane-equiintegrable) if for every $\varepsilon>0$ there is a $\delta: I_{0} \mapsto(0,+\infty)$ such that

$$
\left\|(D) \sum f(\xi) \mu(I)-\int_{I_{0}} f\right\|<\varepsilon
$$

for every $\delta$-fine $M$-partition $D=\{(I, \xi)\}$ of $I_{0}$ and every $f \in K$.

DEFINITION 3. $f: I_{0} \mapsto X$ is called (strongly) measurable if there is a sequence of simple functions $\left(f_{n}\right)$ with $\lim _{n \rightarrow \infty}\left\|f_{n}(t)-f(t)\right\|=0$ for almost all $t \in I_{0}$.

$f: I_{0} \mapsto X$ is called weakly measurable if for each $x^{*} \in X^{*}$ the real function $x^{*}(f): I_{0} \mapsto R$ is measurable.

Two functions $f, g: I_{0} \mapsto X$ are called weakly equivalent on $I_{0}$ if for every $x^{*} \in X^{*}$ the relation

$$
x^{*}(f(t))=x^{*}(g(t))
$$

holds for almost all $t \in I_{0}$.

THEOREM 4. If $f: I_{0} \rightarrow X$ is McShane integrable on $I_{0}$, then

(a) for each $x^{*}$ in $X^{*}, x^{*}(f)$ is McShane integrable on $I_{0}$ and $\int_{I_{0}} x^{*}(f)=x^{*}\left(\int_{I_{0}} f\right)$, (b) $\left\{x^{*}(f) ; x^{*} \in B\left(X^{*}\right)\right\}$ is $M$-equiintegrable on $I_{0}$,

(c) $f$ is weakly measurable.

Proof. Since $f: I_{0} \rightarrow X$ is McShane integrable on $I_{0}$, to every $\varepsilon>0$ there exists a positive function $\delta$ defined on $I_{0}$ such that for any $\delta$-fine $M$-partition $D=\{(I, \xi)\}$ we have $\left\|(D) \sum f(\xi) \mu(I)-\int_{I_{0}} f\right\|<\varepsilon$.

Hence for any $x^{*} \in X^{*}$ we have

$$
\left|(D) \sum x^{*}(f(\xi)) \mu(I)-x^{*}\left(\int_{I_{0}} f\right)\right| \leq\left\|x^{*}\right\|\left\|(D) \sum f(\xi) \mu(I)-\int_{I_{0}} f\right\|<\left\|x^{*}\right\| \varepsilon
$$

for any $\delta$-fine $M$-partition $D=\{(I, \xi)\}$.

Therefore (a) holds. If $x^{*} \in B\left(X^{*}\right)$, then the above inequality gives

$$
\left|(D) \sum x^{*}(f(\xi)) \mu(I)-x^{*}\left(\int_{I_{0}} f\right)\right|<\varepsilon
$$

for every $x^{*} \in B\left(X^{*}\right)$, so the set $\left\{x^{*}(f) ; x^{*} \in B\left(X^{*}\right)\right\}$ is $M$-equiintegrable on $I_{0}$ and $f$ is weakly measurable because for every $x^{*} \in X^{*}$ the real function $x^{*}(f)$ is Lebesgue integrable (see e.g. [5]).

Some other properties of the McShane integral can be found in [2]. 


\section{The relation between the McShane integral and the Pettis integral}

First of all the basic definitions concerning the Pettis integral have to be given. (See e.g. [3], [5], etc.)

DEFINITION 5. If $f: I_{0} \mapsto X$ is weakly measurable such that $x^{*}(f) \in \mathcal{L}$ for all $x^{*} \in X^{*}$, then $f$ is called Dunford integrable . The Dunford integral of $f$ over $E \in \Sigma$ is defined by the element $x_{E}^{* *}$ of $X^{* *}$ such that

$$
x_{E}^{* *}\left(x^{*}\right)=\int_{E} x^{*}(f)
$$

for all $x^{*} \in X^{*}$, and we write $x_{E}^{* *}=(D) \int_{E} f$. By $\mathcal{D}$ the set of all Dunford integrable functions will be denoted.

In the case that $\int_{E} f \in X$ for each $E \in \Sigma$, then $f$ is called Pettis integrable and we write $(P) \int_{E} f$ instead of $(D) \int_{E} f$ to denote the Pettis integral of $f$ over $E \in \Sigma$. Denote by $\mathcal{P}$ the set of all Pettis integrable functions.

We have clearly $\mathcal{P} \subset \mathcal{D}$.

We denote by $\mathcal{L}$ the set of Lebesgue integrable real functions on $I_{0}$ (with respect to the Lebesgue measure $\mu$ ). It should be noted at this point that a real function $f$ belongs to $\mathcal{L}$ if and only if it is McShane integrable, i.e. $\mathcal{L}=\mathcal{M}$ (see [10], [8], etc.).

We use the notation $\mu(E)$ for the Lebesgue measure of a (Lebesgue) measurable set $E \subset I_{0}$.

DEFINITION 6. A set $K \subset \mathcal{L}$ is called uniformly integrable if

$$
\lim _{\mu(E) \rightarrow 0} \int_{E}|f|=0
$$

uniformly for $f \in K$, where $E \subset I_{0}$ are measurable sets.

Since $\left(I_{0}, \Sigma, \mu\right)$ is a finite perfect measure space we can use the result of Theorem 6 from $[4,2]$ in the following form.

THEOREM 7. The function $f: I_{0} \mapsto X$ is Pettis integrable if and only if there is a sequence $\left(f_{n}\right)$ of simple functions from $I_{0}$ into $X$ such that

(a) the set $\left\{x^{*}\left(f_{n}\right) ; x^{*} \in B\left(X^{*}\right), n \in N\right\}$ is uniformly integrable, and

(b) for each $x^{*}$ in $X^{*}, \lim _{n \rightarrow \infty} x^{*}\left(f_{n}\right)=x^{*}(f)$ a.e. on $I_{0}$.

R. A. Gordon proved (see [1], Theorem 17) the following.

THEOREM 8. Let $f: I_{0} \mapsto X$ be given where either $f$ is measurable or $X$ is separable.

If $f$ is Pettis integrable on $I_{0}$, then $f$ is McShane integrable on $I_{0}$. 
REMARK 9. In fact Gordon proved the result given in Theorem 8 for the case when $I_{0}=[a, b] \in R$ is a one-dimensional interval. Looking at [1] it can be seen that the approach of Gordon can be adopted also for the case of a compact interval $I_{0}$ in $R^{m}$.

DEFINITION 10. We say that a Banach space $X$ has the property (P) if there exists a sequence $\left\{x_{m}^{*} \in B\left(X^{*}\right) ; m \in N\right\}$ such that for each $x^{*} \in X^{*}$ there exists a subsequence $\left\{x_{k}^{*} \in B\left(X^{*}\right) ; k \in N\right\}$ of $\left\{x_{m}^{*} \in B\left(X^{*}\right) ; m \in N\right\}$ such that

$$
x_{k}^{*}(x) \rightarrow x^{*}(x) \text { for every } x \in X \text { if } k \rightarrow \infty \text {. }
$$

In [2] the following was proved.

THEOREM 11. If $f: I_{0} \mapsto X$ is Pettis integrable on $I_{0}, f$ is weakly equivalent to a measurable function $g: I_{0} \mapsto X$ and $X$ has the property $(P)$ then $f$ is McShane integrable on $I_{0}$, i.e. $\mathcal{P} \subset \mathcal{M}$.

G. F. Stefánsson in [11] proved the following.

PROPOSITION 12. All weakly measurable functions determined by reflexive spaces are weakly equivalent to strongly measurable functions.

Let us recall that a weakly measurable function $f: I_{0} \mapsto X$ is said to be determined by a subspace $D$ of $X$ if for $x^{*} \in X^{*}$ which is restricted to $D$ equals zero $\left(\left.x^{*}\right|_{D}=0\right)$ the function $x^{*}(f)$ equals zero almost everywhere on $I_{0}$.

Since every weakly measurable function $f: I_{0} \mapsto X$ is determined by the space $X$ itself, we conclude easily that the following holds.

PROPOSITION 13. If the Banach space $X$ is reflexive and $f: I_{0} \mapsto X$ is weakly measurable, then there exists a strongly measurable $g: I_{0} \mapsto X$ which is weakly equivalent to $f$.

Using this and Theorem 11 we obtain.

COROLLARY 14. If the Banach space $X$ is reflexive with the property $(P)$ and $f: I_{0} \mapsto X$ is Pettis integrable, then $f$ is McShane integrable on $I_{0}$, i.e. $\mathcal{P} \subset \mathcal{M}$.

REMARK 15. Stefánsson's Proposition 12 can be used for stating the following.

If $f: I_{0} \mapsto X$ is Pettis integrable on $I_{0}, f$ is determined by a reflexive space and $X$ has the property $(P)$, then $f$ is McShane integrable on $I_{0}$.

THEOREM 16. If $f$ is McShane integrable on $I_{0}$, then $f$ is Pettis integrable, i.e., $\mathcal{M} \subset \mathcal{P}$.

The proofs of the above results can be found in [2]. 
From Theorem 16 and by the properties of Pettis integrable functions presented in [3] (Theorem 5, p. 53; Corollary 9, p. 56) we get the following.

COROLLARY 17. If $f: I_{0} \mapsto X$ is McShane integrable on $I_{0}$, then

(1) $F: E \mapsto \int_{E} f d \mu$ is a countable additive $\mu$-continuous vector measure on $\Sigma$,

(2) $\{F(E) ; E \in \Sigma\}$ is bounded,

(3) $F(\Sigma)$ is relatively weakly compact.

By Corollary 14 and Theorem 16 we also have the following.

THEOREM 18. Let the Banach space $X$ be reflexive with the property $(P)$. Then $f:[a, b] \mapsto X$ is McShane integrable on $[a, b]$ if and only if $f$ is Pettis integrable, i.e., $\mathcal{M}=\mathcal{P}$.

THEOREM 19. Let $f: I_{0} \mapsto X$ be weakly equivalent to a measurable function $g: I_{0} \mapsto X$ and $X$ has the property $(P)$. Then the following statements are equivalent.

(a) $f$ is McShane integrable on $I_{0}$,

(b) $f$ is Pettis integrable on $I_{0}$,

(c) there is a sequence $\left(f_{n}\right)$ of simple functions from $I_{0}$ into $X$ such that the set $\left\{x^{*}\left(f_{n}\right) ; x^{*} \in B\left(X^{*}\right), n \in N\right\}$ is uniformly integrable and for each $x^{*}$ in $X^{*}$ we have $\lim _{n \rightarrow \infty} x^{*}\left(f_{n}\right)=x^{*}(f)$ a.e. on $I_{0}$,

Proof. By Theorem 11 and Theorem 16, we know that (a) and (b) are equivalent and (b) is equivalent to (c) by Theorem 7 . $[3])$.

Let us denote by $\mathcal{B}$ the set of all Bochner integrable functions $f: I_{0} \mapsto X$ (see e.g.

In our paper [6] we gave some characterizations of $\mathcal{B}$ and we proved among others the following.

THEOREM 20. The inclusion $\mathcal{B} \subset \mathcal{M}$ holds in general and $\mathcal{B}=\mathcal{M}$ if and only if the Banach space $X$ is finite dimensional.

Since finite dimensional Banach spaces are separable, we can combine this with Theorem 8 and Theorem 20 to obtain the following.

THEOREM 21. The inclusion $\mathcal{B} \subset \mathcal{P}$ holds in general and we have $\mathcal{B}=\mathcal{P}$ if and only if the Banach space $X$ is finite dimensional.

\section{Relation between the McShane integral and the Pettis integral}

In this section we define the weak McShane integral and discuss the relation between the weak McShane integral and the Pettis integral. 
DEFINITION 22. An $X$-valued function $f$ is said to be weakly McShane integrable on $I_{0}$ if for every $x^{*} \in X^{*}$ the real function $x^{*}(f)$ is McShane integrable on $I_{0}$ and for every interval $I \subset I_{0}$, there is a $x_{I} \in X$ such that $\int_{I} x^{*}(f)=x^{*}\left(x_{I}\right)$. We write $X_{I}=(W M) \int_{I} f$.

$f$ is weakly McShane integrable on a set $E \subseteq I_{0}$ if the function $f \cdot \chi_{E}$ is weakly McShane integrable on $I_{0}$, where $\chi_{E}$ denotes the characteristic function of $E$. $f$ on $E$.

We write $(W M) \int_{E} f=(W M) \int_{I_{0}} f \chi_{E}=F(E)$ for the weak McShane integral of

Denote the set of all weakly McShane integrable functions $f: I_{0} \mapsto X$ by $\mathcal{W M}$.

Looking at the corresponding definitions and using the well-known fact that the McShane integrability of the real function $x^{*}(f)$ is equivalent to its Lebesgue integrability, we obtain immediately the following statement concerning the various sets of integrable functions.

PROPOSITION 23. If $X$ is an arbitrary Banach space then

$$
\mathcal{M} \subset \mathcal{P} \subset \mathcal{W M} \subset \mathcal{D}
$$

It is easy to prove that the weak McShane integral has the following basic properties.

THEOREM 24. Let $f$ and $g$ be functions mapping $I_{0}$ into $X$.

(a) If $f$ is weakly McShane integrable on $I_{0}$, then $f$ is weakly McShane integrable on every subinterval of $I \subset I_{0}$.

(b) If $f$ is weakly McShane integrable on each of the intervals $I_{1}$ and $I_{2}$, where $I_{i}$ are non-overlapping and $I_{1} \cup I_{2}=I$ is an interval, then $f$ is weakly McShane integrable on $I$ and

$$
(W M) \int_{I} f=(W M) \int_{I_{1}} f+(W M) \int_{I_{2}} f
$$

(c) If $f$ and $g$ are weakly McShane integrable on $I_{0}$ and if $\alpha$ and $\beta$ are real numbers, then $\alpha f+\beta g$ is weakly McShane integrable on $I_{0}$ and

$$
(W M) \int_{I_{0}}(\alpha f+\beta g)=\alpha(W M) \int_{I_{0}} f+\beta(W M) \int_{I_{0}} g .
$$

(d) If $f=g$ almost everywhere (with respect to the Lebesgue measure $\mu$ in $R^{m}$ ) on $I_{0}$ and $f$ is weakly McShane integrable, then $g$ is weakly McShane integrable on $I_{0}$ and $(W M) \int_{I_{0}} f=(W M) \int_{I_{0}} g$.

Let us start with an example which is a modified version of an example of Gordon from [7]. 
EXAMPLE 25. A weakly McShane integrable function that not Pettis integrable.

For each $n \in N$ let

$$
I_{n}^{\prime}=\left(\frac{1}{n+1}, \frac{n+\frac{1}{2}}{n(n+1)}\right), I_{n}^{\prime \prime}=\left(\frac{n+\frac{1}{2}}{n(n+1)}, \frac{1}{n}\right)
$$

and define $f_{n}:[0,1] \rightarrow R$ by

$$
f_{n}(t)=2 n(n+1)\left(\chi_{I_{n}^{\prime}}(t)-\chi_{I_{n}^{\prime \prime}}(t)\right) .
$$

Then the sequence $\left\{f_{n}\right\}$ converges to 0 pointwise and $\left\{\int_{I} f_{n}\right\}$ converges to 0 , i.e. $\left\{\int_{I} f_{n}\right\} \in c_{0}$ for each interval $I$. Define $f:[0,1] \rightarrow c_{0}$ by $f(t)=\left\{f_{n}(t)\right\}$ for $t \in[0,1]$.

Let $x^{*}=\left\{\alpha_{n}\right\} \in l_{1}=\left(c_{0}\right)^{*}$. Then $x^{*}(f(t))=\sum_{n} \alpha_{n} f_{n}(t)$ for $t \in[0,1]$. Since

$$
\sum_{n} \int_{0}^{1}\left|\alpha_{n} f_{n}\right|=\sum_{n} 2\left|\alpha_{n}\right|<+\infty
$$

the Levi monotone convergence theorem applies to show that $x^{*}(f)$ is Lebesgue integrable and, naturaly, also McShane integrable on $[0,1]$ and

$$
(M) \int_{0}^{1} x^{*}(f)=\sum \int_{0}^{1} \alpha_{n} f_{n}=\sum \alpha_{n} \int_{0}^{1} f_{n}=x^{*}\left(\left\{\int_{0}^{1} f_{n}\right\}\right)=0
$$

because $\int_{0}^{1} f_{n}=0$ for every $n \in N$.

If $I \subset[0,1]$ is an interval, then we have $\int_{I} f=\left\{\int_{I} f_{n}\right\} \in c_{0}$ by the choice of $\left\{f_{n}\right\}$ and $\int_{I} x^{*}(f)=x^{*}\left(\left\{\int_{I} f_{n}\right\}\right)$.

This yields that $f$ is weakly McShane integrable on $[0,1]$.

On the other hand, $f$ is not Pettis integrable on $[0,1]$ since for the measurable set $E=\cup_{n} I_{n}^{\prime}$ we have $\int_{E} f=\left\{\int_{E} f_{n}\right\}=\{1\} \in l_{\infty} \backslash c_{0}$.

According to Proposition 23, this example shows also that the function $f:[0,1] \rightarrow$ $c_{0}$ given above cannot be McShane integrable.

We come now to our main result.

THEOREM 26. Suppose that $X$ is a Banach space and that a function $f: I_{0} \rightarrow X$ is given. Then the weak McShane integral and the Pettis integral of the function $f$ are equivalent if and only if $X$ contains no copy of $c_{0}$.

Proof. If the weak McShane integral and the Pettis integral are equivalent, according to Example 25, it is easy to see that $X$ cannot contain a copy of $c_{0}$.

Conversely, assume that $f$ is weakly McShane integrable on $I_{0}$ and $X$ contains no copy of $c_{0}$.

Suppose that $E$ is a measurable subset of $I_{0}$. 
Given a $\lambda$ such that $0<\lambda<1$ an interval $I$ in $R^{m}$ is called $\lambda$-regular if

$$
r(I)=\frac{\mu(I)}{[d(I)]^{m}}>\lambda,
$$

$(r(I)$ is the regularity of the interval $I)$ and $d(I)=\sup \{|x-y| ; x, y \in I\},|x-y|=$ $\max \left\{\left|x_{1}-y_{1}\right|, \ldots,\left|x_{m}-y_{m}\right|\right\}$, and $x=\left(x_{1}, \ldots, x_{m}\right), y=\left(y_{1}, \ldots, y_{m}\right)$.

Let $\left\{\varepsilon_{k}\right\}$ be a decreasing sequence of positive reals tending to zero.

Let $\delta_{k}(t)>\delta_{k+1}(t) \rightarrow 0$ for $k \rightarrow \infty$ and let $0<\lambda<1$ be fixed.

Set

$$
\Phi_{n}=\left\{I \subset I_{0}, I \text { an interval; } t \in I \subset B\left(t, \delta_{n}(t)\right), r(I)>\lambda, t \in E\right\} .
$$

Then $\Phi=\left\{\Phi_{n} ; n=1,2, \ldots\right\}$ is a Vitali cover of $E$.

By the Vitali covering theorem (see e.g. [9], Proposition 9.2.4), there is a sequence $E_{n}$ ( $E_{n}$ is the finite union of non-overlapping intervals belonging to $\Phi$ ) such that

$$
\mu\left(E \backslash E_{n}\right)<\varepsilon_{n},
$$

i.e., $\mu\left(E \backslash E_{n}\right) \rightarrow 0$ for $n \rightarrow \infty$ for $n \rightarrow \infty$.

Hence there exists a sequence $\left\{I_{n}\right\}$ of non-overlapping intervals $I_{n}$ such that $\mu(E \backslash$ $\left.\cup_{n} I_{n}\right)=0$. Because $f$ is weakly McShane integrable, the real function $x^{*}(f)$ is Lebesgue integrable and $(W M) \int_{I_{n}} f \in X$ for all $n \in N$.

Thus, for each $x^{*} \in X^{*}, \int_{E \backslash \cup_{n} I_{n}} x^{*}(f)=0$ and

$$
\begin{gathered}
\int_{E} x^{*}(f)=\int_{E \backslash \cup_{n} I_{n}} x^{*}(f)+\int_{\cup_{n} I_{n}} x^{*}(f)=\int_{\cup_{n} I_{n}} x^{*}(f)= \\
=\sum_{n} \int_{I_{n}} x^{*}(f)=\sum_{n} x^{*}\left((W M) \int_{I_{n}} f\right)=\lim _{N \rightarrow \infty} \sum_{n=1}^{N} x^{*}\left((W M) \int_{I_{n}} f\right) .
\end{gathered}
$$

Since $X$ contains no copy of $c_{0}$, by the Bessaga-Pelczynski Theorem ([3], p. 22) the series $\sum_{n}(W M) \int_{I_{n}} f$ is unconditionally convergent in norm to an element $x_{E} \in X$, i.e.

$$
\lim _{N \rightarrow \infty}\left\|\sum_{n=1}^{N}(W M) \int_{I_{n}} f-x_{E}\right\|=0
$$

Since

$$
x^{*}\left(\sum_{n=1}^{N}(W M) \int_{I_{n}} f-x_{E}\right)=\sum_{n=1}^{N} x^{*}\left((W M) \int_{I_{n}} f\right)-x^{*}\left(x_{E}\right) \rightarrow 0 \text { for } N \rightarrow \infty
$$

we obtain

$$
\int_{E} x^{*}(f)=\lim _{N \rightarrow \infty} x^{*}\left(\sum_{n=1}^{N}(W M) \int_{I_{n}} f\right)=x^{*}\left(x_{E}\right)
$$


and by definition $f$ is Pettis integrable on $I_{0}$ and $(W M) \int_{I_{0}} f=(P) \int_{I_{0}} f$.

Using Theorem 19 and Theorem 26 we obtain immediately

COROLLARY 27. If $f: I_{0} \mapsto X$ is weakly equivalent to a measurable function $g: I_{0} \mapsto X$ and $X$ has the property $(P)$ then the McShane and the weak McShane integral are equivalent if and only if $X$ does not contain a copy of $c_{0}$.

Acknowledgement: The first author was supported as a postdoctoral fellow by the People's Republic of China in the frame of the exchange programme between the Ministries of Education of the People's Republic of China and the Czech Republic. The second author was supported by grant No. 201/01/1199 of the GA of the Czech Republic.

\section{REFERENCES}

[1] Gordon, R. A.: The McShane integral of Banach-valued functions, Illinois Journal of Math., 34, (1990), 557-567.

[2] Schwabik, Š. and Ye, G.: The McShane integral and the Pettis integral of Banach space-valued functions defined on $R^{m}$, submitted to the Illinois J. of Math.

[3] Diestel, J. and Uhl, J. J. JR.: Vector Measures, Math. Surveys, No.15, Amer. Math. Soc., Providence, R.I., 1977.

[4] Geitz, R. F.: Pettis integration, Proc. of the Amer. Math. Soc., 82, (1981), 81-86.

[5] Musial, K.: Topics in the theory of Pettis integration, Rend. Ist. Mat. Univ. Trieste, 23, (1991), 177-262.

[6] Schwabik, Š. and YE, G.: On the strong McShane integral of functions with values in a Banach space, Czech. Math. Journal, to appear.

[7] Gordon, R. A.: The Denjoy extension of the Bochner, Pettis, and Dunford integrals, Studia Mathematica, T.XCII (1989), 73 - 91.

[8] Lee Peng YeE: Lanzhou Lectures on Henstock Integration, World Scientific, Singapore, 1989.

[9] Pfeffer, W.F.: The Riemann approach to integration: Local geometric theory, Cambridge University Press, 1993.

[10] Gordon, R. A.: The integrals of Lebesgue, Denjoy, Perron, and Henstock, American Mathematical Society, 1994.

[11] Stefánsson, G. S.: Pettis integrability, Transactions of the AMS, 330, (1992), 401-418. 